Check for updates

Cite this: Chem. Commun., 2019, 55,7319

Received 12th April 2019, Accepted 29th May 2019

DOI: $10.1039 / \mathrm{c} 9 \mathrm{cc} 02861 \mathrm{~g}$

rsc.li/chemcomm

\section{Upcycling a plastic cup: one-pot synthesis of lactate containing metal organic frameworks from polylactic acid $\dagger$}

\author{
Benjamin Slater, (D) ${ }^{\text {ab }}$ So-On Wong, ${ }^{a}$ Andrew Duckworth, ${ }^{a}$ Andrew J. P. White, (D) \\ Matthew R. Hill (D) ${ }^{b}$ and Bradley P. Ladewig (D) *ad
}

\begin{abstract}
Waste PLA can be upcycled to metal organic frameworks of potential high value in a one-pot synthesis scheme, where PLA depolymerisation occurs in situ. Three homochiral lactate based frameworks were successfully synthesised and characterised from PLA as a feed source, including ZnBLD. The chiral separation ability of ZnBLD was maintained.
\end{abstract}

Chirality is a phenomenon present throughout nature, for example the chiral, helical structure of DNA. ${ }^{1}$ Enantiopure chemicals are required on industrial scales for a wide variety of industries such as pharmaceuticals, where one enantiomer of a drug may be metabolised differently to its mirror image. ${ }^{2}$ Metal Organic Frameworks (MOFs) are a series of porous coordination polymers consisting of metal nodes coordinated to organic 'linker' ligands. ${ }^{3}$ MOFs have several promising applications from sensing, ${ }^{4}$ to separations, ${ }^{5,6}$ including chiral separations. ${ }^{7,8}$ A significant number of reported MOFs are synthesised from terephthalic acid as an organic linker. ${ }^{9,10}$ A search of the Cambridge Structural Database (Conquest) for all structures containing 1,4-benzenedicarboxylate revealed 2028 reported structures, of which a very high proportion are MOFs. ${ }^{11,12}$ Polyethylene terephthalate is a polyester consisting of terephthalic acid and ethylene glycol in a 1:1 molar ratio. Research efforts have focused on uses for excess PET in the environment, and alternative recycling methods, for example, utilisation of PET in asphalt pavement, ${ }^{13}$ and biological based recycling. ${ }^{14}$ This is likely to have been influenced by growing concerns about the environmental impact of plastic where it is estimated that as of 2015,8300 million

\footnotetext{
${ }^{a}$ Barrer Centre, Department of Chemical Engineering, Imperial College London, London SW7 2AZ, UK. E-mail: Bradley.ladewig@imperial.ac.uk

${ }^{b}$ CSIRO, Private Bag 10, Clayton South MDC, Victoria 3169, Australia

${ }^{c}$ Department of Chemistry, Imperial College London, Molecular Sciences Research Hub, White City Campus, London, W12 OBZ, UK

${ }^{d}$ Institute for Micro Process Engineering, Karlsruhe Institute of Technology, 76344 Eggenstein-Leopoldshafen, Germany

$\dagger$ Electronic supplementary information (ESI) available: Experimental details and additional results and discussion. CCDC 1908241. Raw XRD spectra and other supporting data are available from the open data repository: https://doi.org/10. 5281/zenodo.2638093. For ESI and crystallographic data in CIF or other electronic format see DOI: $10.1039 / \mathrm{c} 9 \mathrm{cc} 02861 \mathrm{~g}$
}

metric tons of virgin plastic was produced, $76 \%$ of which is now waste. ${ }^{15}$ Recently, PET has been used as a terephthalic acid source for the synthesis of several MOFs. PET was successfully depolymerised under microwave irradiation before being used as a linker source in solvothermal synthesis of two terephthalate based MOFs. ${ }^{16}$ Subsequently, reports highlighted the synthesis of several MOFs directly from PET as terephthalate source (without a prior depolymerisation step), utilising either microwave, ${ }^{17}$ or conventional heating techniques. ${ }^{18,19}$ This process of upcycling waste polymer receptacles such as PET bottles and cups, has the potential to be applied to other waste polymer materials for the synthesis of MOFs. ZnBLD [ $\mathrm{Zn}_{2}(\mathrm{bdc})(\mathrm{L}$-lactate)(dmf)].(DMF) (bdc $=1,4$-benzenedicarboxylate), $(\mathrm{dmf}=N, N$-dimethylformamide) is a homochiral MOF synthesised from zinc nitrate hexahydrate, terephthalic and L-lactic acids in a 2:1:1 molar ratio respectively. ${ }^{7}$ Hypothetically, ZnBLD could be synthesised from PET as terephthalic acid source and polylactic acid (PLA) as the lactic acid source. MOFs $1201\left[\mathrm{Ca}_{14}(\mathrm{~L} \text {-lactate })_{20}(\text { acetate })_{8}\left(\mathrm{C}_{2} \mathrm{H}_{5} \mathrm{OH}\right)\left(\mathrm{H}_{2} \mathrm{O}\right)\right]$ and 1203 $\left[\mathrm{Ca}_{6}(\mathrm{~L}-\mathrm{lactate})_{3}(\text { acetate })_{9}\left(\mathrm{H}_{2} \mathrm{O}\right)\right]$ are synthesised from calcium acetate and L-lactic acid in a 1:2 and 1:1 molar ratio respectively. ${ }^{20}$ In addition to ZnBLD, there is the possibility that these frameworks can be synthesised from PLA as the lactic acid source. Schemes S1 and S2 (ESI $\dagger$ ) depict the depolymerisation schemes for PET and PLA to the corresponding monomers. PLA is also a polyester formed from the polymerisation of lactic acid. In the polymerisation process of both PLA and PET, one water molecule is removed in each ester linkage formed ( 1 mole of water per mole of lactic acid/1 mole of water per mole of terephthalic acid). Therefore, the minimum molar quantity of water must be present in the 1-pot polymer based MOF synthesis procedures in order to successfully depolymerise the polymers.

PLA is commonly found in biodegradable single use plastic items such as plastic cups. Although it is often considered as a recyclable polymer, PLA waste is typically treated by methods such as composting, hydrolysis and natural decomposition. ${ }^{21}$ Pure lactic acid is stable as a solid below its melting point of $16.8{ }^{\circ} \mathrm{C},{ }^{22}$ but under standard conditions will melt and absorb water. Consequently, the price of pure L-lactic acid (required for 

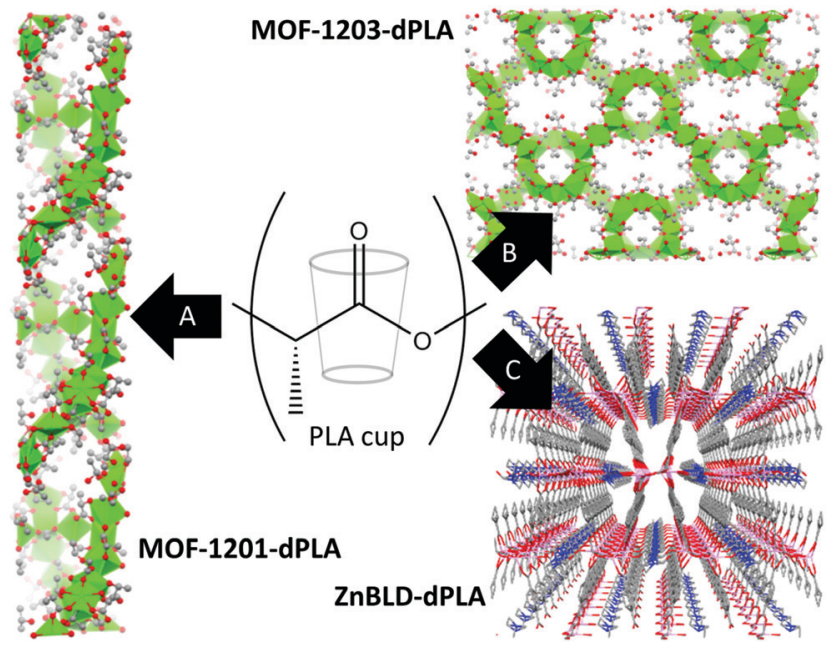

Scheme 1 Synthesis of three homochiral lactate based MOFs from PLA cups and single crystal X-ray diffraction structures of: (A) chiral channels of MOF-1201 viewed along the $b$ axis, (B) view along the $a$ axis of MOF-1203 and (C) view along the $a$ axis of ZnBLD-dPLA, (perspective used for artistic effect), $\mathrm{Ca}$ and $\mathrm{Ca}^{2+}$ polyhedra in green, $\mathrm{O}$ in red, $\mathrm{C}$ in grey, $\mathrm{N}$ in blue and $\mathrm{Zn}$ in pink. $\mathrm{H}$ atoms have been omitted for clarity. ${ }^{20}$

the synthesis of ZnBLD and MOFs 1201 and 1203) is considerably more expensive than the more stable but lower purity aqueous solution. $\$$ Other studies have previously reported the synthesis of PLA-MOF composites for disposable electronic applications, ${ }^{23}$ but, to the best of our knowledge, PLA has not been used as lactic acid ligand source for MOF preparation.

From herein, we report the synthesis and chiral separation properties of ZnBLD, and the syntheses of MOF-1201 and MOF1203 by replacing L-lactic acid with the equivalent molar ratio of PLA illustrated in Scheme 1. All of these frameworks have a molar excess of water from the metal salts (calcium acetate monohydrate and zinc nitrate hexahydrate). However, ZnBLD is synthesised from DMF with no requirement for this to be anhydrous. As DMF is hygroscopic, having DMF seasoned by atmospheric water vapour can significantly increase the water content. Contrastingly, MOFs 1201 and 1203 require anhydrous ethanol and methanol respectively. Therefore, it is expected that PLA will show considerably different depolymerisation behaviours in each solvent. Table S1 (ESI $\dagger$ ) shows the composition of the frameworks synthesised based on the adjusted molar ratios for the PLA synthesised MOFs. The suffix dPLA is used to describe MOFs synthesised from waste PLA (dPLA = depolymerised polylactic acid). Additional, unsuccessful attempts were made to synthesise ZnBLD from waste PET, this is most likely because hydrolysis of PET requires higher temperatures (above $200^{\circ} \mathrm{C}$ ) than the synthesis temperature of $\mathrm{ZnBLD}\left(110{ }^{\circ} \mathrm{C}\right)$ and is typically performed in aqueous media, ${ }^{24}$ this is outlined in the ESI. $\dagger$ The synthesised frameworks are characterised and compared with their counterparts synthesised from pure lactic acid and finally, the enantioseparation ability and guest molecule loading of ZnBLDdPLA is compared with ZnBLD.

The dPLA frameworks powder X-ray diffraction (PXRD) traces were recorded and compared with the predicted and experimental

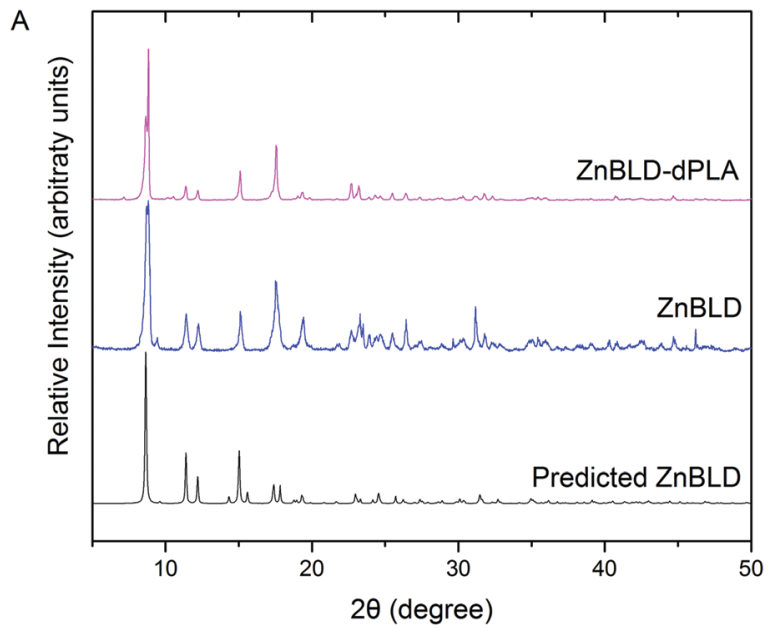

B

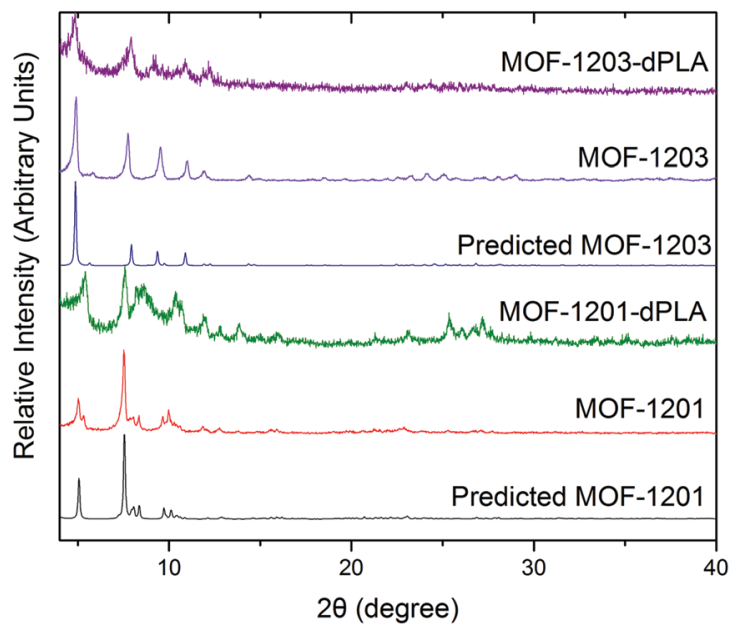

Fig. 1 Experimental and predicted powder X-ray diffraction traces of: (A) ZnBLD and ZnBLD-dPLA and (B) MOFs 1201 and 1203 and MOFs $1201-$ dPLA and 1203-dPLA.

patterns using pure s-lactic acid as feed source, to confirm phase purity, these results are displayed in Fig. 1. The phases of ZnBLD and MOFs 1201 and 1203 were obtained. There were slight variations in the PXRD traces of ZnBLD and ZnBLD-dPLA. The relative intensity of the $\mathrm{Zn}(\mathrm{bdc}) \cdot x \mathrm{H}_{2} \mathrm{O}\left(8.6^{\circ} 2 \theta\right)$ phase (caused by partial hydrolysis of the framework), had a relatively higher intensity in the ZnBLD-dPLA crystals. We suspected that this was caused by a higher concentration of this phase in the ZnBLD-dPLA crystals than that of ZnBLD, which may be a consequence of using PLA as feed source instead of L-lactic acid. In order to ensure consistency with our results, the same batch of as-synthesised crystals were used for all characterisation and separation experiments. The PXRD traces of MOFs 1201-dPLA and 1203-dPLA were less crystalline than the pure lactic acid synthesised counterparts. Additionally, the product was obtained as a mixture with a gel like by-product, which is commonly prepared from calcium acetate and alcohol. The crystal structure of ZnBLD-dPLA is reported in the ESI, $\dagger$ this structure is isostructural with ZnBLD (previously reported) with the same unit cell and space group $\left(P 2_{2} 2_{1} 2_{1}\right){ }^{7}$ 


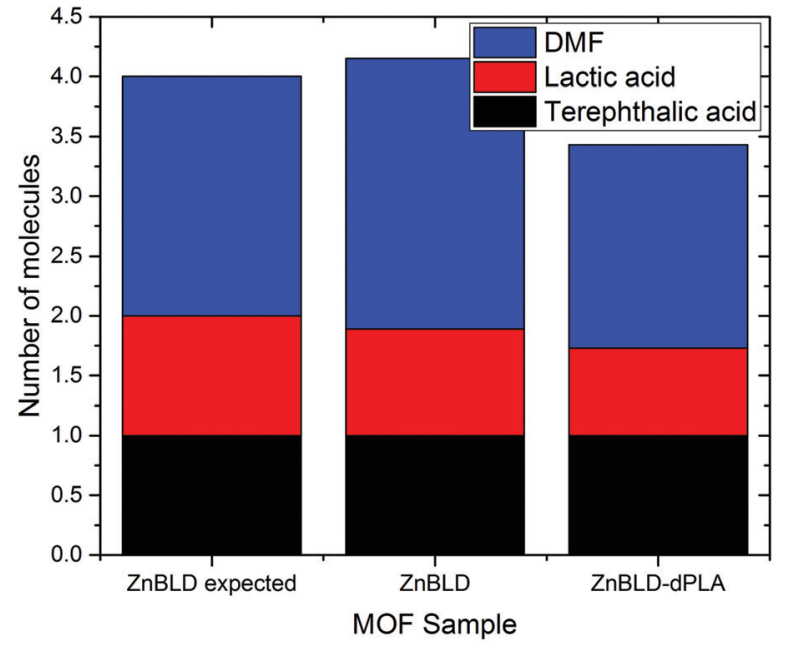

Fig. 2 Organic compositions of ZnBLD and ZnBLD-dPLA determined by NMR spectroscopy.

As-synthesised MOF crystals of ZnBLD, MOFs 1201 and 1203 and their dPLA counterparts were digested in DMSO- $\mathrm{d}_{6} / \mathrm{DCl}$ before nuclear magnetic resonance (NMR) spectra were recorded to determine the organic composition of the frameworks which could be compared with the expected formulas. Comparing the spectra of ZnBLD and ZnBLD-dPLA, (Fig. 2) there was a slight difference in the ratio of terephthalic acid:lactic acid (1:0.89 and 0.73 respectively). We can therefore conclude that there is a higher concentration of $\mathrm{Zn}(\mathrm{bdc}) \cdot x \mathrm{H}_{2} \mathrm{O}$ in ZnBLD-dPLA as observed in the PXRD traces.

As-synthesised MOFs 1201 and 1203 produced consistent organic compositions with the expected formulas for these MOFs but the dPLA synthesised counterparts contained higher concentrations of acetate and solvent (Fig. S1 and S2, ESI $\dagger$ ). This is caused by the formation of the amorphous gel like phase.

The chiral separation ability of ZnBLD-(dPLA) was compared with ZnBLD for four racemates (1-phenylethanol, 2-butanol, limonene and 2-methyl-2,4-pentanediol) using an adsorption, desorption method. Whereby, each framework was soaked in neat solutions of the racemates, filtered and soaked in dichloromethane to desorb the adsorbed guest species. Subsequent analysis of the supernatant by chiral gas chromatography revealed the enantiomeric excess of the separation process, and using Table S3 (ESI $\dagger$ ), the preferentially adsorbed enantiomers can be determined. Fig. 3 shows that the separation ability of the dPLA synthesised frameworks were not negatively impacted by the modified synthesis method for the racemates tested. Interestingly, there was a significant increase in the enantiomeric excess for the limonene separation when using the ZnBLD-dPLA framework. The ZnBLD 1-phenylethanol and 2-butanol enantiomeric excess values are previously reported. ${ }^{25}$ Further investigation is required to better understand the selectivity increase effecting limonene. We hypothesise that this is caused by the reduction in crystal size of ZnBLD-dPLA (see Images S3 and S4, ESI $\dagger$ ). The enantiomeric excess for a mandelate based homochiral MOF has been shown to increase with time from 1-7 days. However, the influence of the crystal size was not taken into consideration in

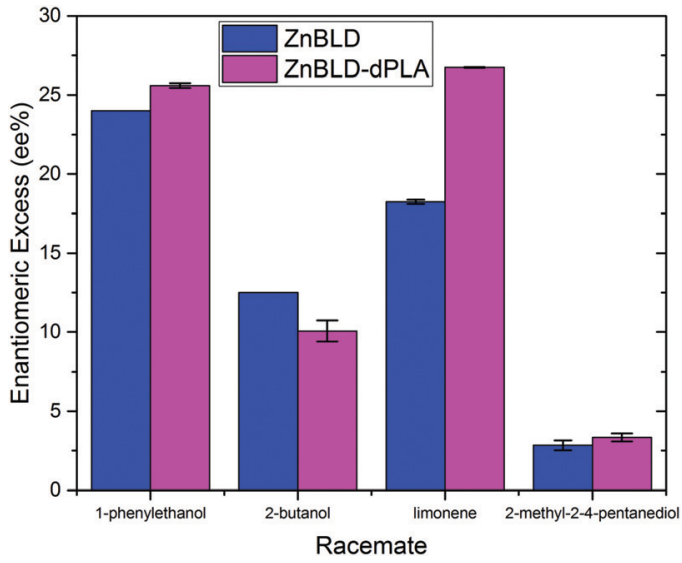

Fig. 3 Enantiomeric excesses obtained from enantioselective adsorption in ZnBLD and ZnBLD-dPLA.

this study, ${ }^{26}$ and to the best of our knowledge, has not been investigated in other studies. Our hypothesis is based on the assumption that smaller crystals will reach maximum separation capacity faster than larger ones.

Additionally the loading of the four adsorbed racemates was determined by ${ }^{1} \mathrm{H}$ NMR spectroscopy in the ZnBLD and ZnBLDdPLA frameworks. In this process, after adsorption of each racemate, the MOF crystals were filtered and subsequently dissolved in DMSO- $\mathrm{d}_{6} / \mathrm{DCl}$ before NMR spectra were recorded. Characteristic proton resonances of the framework terephthalic acid and selected peaks from each racemate were integrated in order to determine the loading (where the loading of racemates was compared with 1 molecule of the framework terephthalic acid), further details are reported in the ESI. $\dagger$ Fig. S5 (ESI $\dagger$ ) displays the loading results from the adsorbed chiral guest molecules. There were slight variations in guest molecule loading when comparing the two frameworks, but overall, neither framework consistently outperformed the other.

We have shown, for the first time that polylactic acid can be utilised as a feed source for the synthesis of lactate containing MOFs such as ZnBLD and MOFs 1201 and 1203. By using this method, we were able to upcycle post-consumer use PLA products into potentially high value materials. We demonstrate that it is possible to bypass the requirement to purchase expensive high purity lactic acid and instead use waste plastic drinking cups. The ZnBLD-dPLA framework upholds the equivalent or enhanced chiral separation properties as its counterpart synthesised from pure chemicals without an overall reduction in loading. This can considerably reduce the cost of manufacturing of these products. We expect that by altering the synthesis of MOFs 1201 and 1203, to increase the PLA depolymerisation efficiency, these MOFs can be synthesised effectively without obtaining the amorphous gel, and with the equivalent crystallinity of the originally reported MOFs as shown for ZnBLD.

BDS acknowledges CSIRO and Imperial College London for financial support, and thanks Dr Jason Hallett for discussions on PLA waste. The authors acknowledge Dr Charles Romain for providing PLA cups. 


\section{Conflicts of interest}

There are no conflicts to declare.

\section{Notes and references}

\$ Prices of lactic acids on the UK Sigma Aldrich website on the 21/02/ 2019: $1 \mathrm{~L} 80 \%$ aqueous solution L-lactic acid = £38.50(GBP), $10 \mathrm{~g} \mathrm{98 \%}$ L-lactic acid $=£ 121(\mathrm{GBP})$.

1 J. D. Watson and F. H. C. Crick, Nature, 1953, 171, 737-738.

2 S. S. Adams, P. Bresloff and C. G. Mason, J. Pharm. Pharmacol., 1976, 28, 256-257.

3 O. M. Yaghi, G. Li and H. Li, Nature, 1995, 378, 703.

4 X. Fang, B. Zong and S. Mao, Nanomicro Lett., 2018, 10, 64.

5 D. Banerjee, C. M. Simon, S. K. Elsaidi, M. Haranczyk and P. K. Thallapally, Chem, 2018, 4, 466-494.

6 S. Mukherjee, A. V. Desai and S. K. Ghosh, Coord. Chem. Rev., 2018, 367, 82-126.

7 D. N. Dybtsev, A. L. Nuzhdin, H. Chun, K. P. Bryliakov, E. P. Talsi, V. P. Fedin and K. Kim, Angew. Chem., Int. Ed., 2006, 45, 916-920.

8 C. Zhuo, Y. Wen, S. Hu, T. Sheng, R. Fu, Z. Xue, H. Zhang, H. Li, J. Yuan, X. Chen and X. Wu, Inorg. Chem., 2017, 56, 6275-6280.

9 G. Férey, C. Mellot-Draznieks, C. Serre, F. Millange, J. Dutour, S. Surblé and I. Margiolaki, Science, 2005, 309, 2040-2042.

10 J. H. Cavka, S. Jakobsen, U. Olsbye, N. Guillou, C. Lamberti, S. Bordiga and K. P. Lillerud, J. Am. Chem. Soc., 2008, 130, 13850-13851.

11 S. R. Miller, P. A. Wright, C. Serre, T. Loiseau, J. Marrot and G. Ferey, Chem. Commun., 2005, 3850-3852, DOI: 10.1039/b506677h.
12 M. Lammert, M. T. Wharmby, S. Smolders, B. Bueken, A. Lieb, K. A. Lomachenko, D. D. Vos and N. Stock, Chem. Commun., 2015, 51, 12578-12581.

13 A. F. Ahmad, A. R. Razali and I. S. M. Razelan, Utilization of polyethylene terephthalate (PET) in asphalt pavement: A review, IOP Publishing Ltd, Bristol, 2017.

14 R. Koshti, L. Mehta and N. Samarth, J. Polym. Environ., 2018, 26, 3520-3529.

15 R. Geyer, J. R. Jambeck and K. L. Law, Sci. Adv., 2017, 3, e1700782. 16 Manju, P. Kumar Roy, A. Ramanan and C. Rajagopal, Mater. Lett., 2013, 106, 390-392.

17 W. P. R. Deleu, I. Stassen, D. Jonckheere, R. Ameloot and D. E. De Vos, J. Mater. Chem. A, 2016, 4, 9519-9525.

18 J. Ren, X. Dyosiba, N. M. Musyoka, H. W. Langmi, B. C. North, M. Mathe and M. S. Onyango, Int. J. Hydrogen Energy, 2016, 41, 18141-18146.

19 S.-H. Lo, D. Senthil Raja, C.-W. Chen, Y.-H. Kang, J.-J. Chen and C.-H. Lin, Dalton Trans., 2016, 45, 9565-9573.

20 J. Yang, C. A. Trickett, S. B. Alahmadi, A. S. Alshammari and O. M. Yaghi, J. Am. Chem. Soc., 2017, 139, 8118-8121.

21 H. Y. Lin, S. Y. Tsai, H. T. Yu and C. P. Lin, J. Polym. Environ., 2018, 26, 122-131.

22 J. G. Stark and H. G. Wallace, Chemistry Data Book, Murray, 1982.

23 X. Shi, X. Dai, Y. Cao, J. Li, C. Huo and X. Wang, Ind. Eng. Chem. Res., 2017, 56, 3887-3894.

24 L. Liu, D. Zhang, L. An, H. Zhang and Y. Tian, J. Appl. Polym. Sci., 2005, 95, 719-723.

25 B. Slater, Z. Wang, S. Jiang, M. R. Hill and B. P. Ladewig, J. Am. Chem. Soc., 2017, 139, 18322-18327.

26 S.-Y. Zhang, L. Wojtas and M. J. Zaworotko, J. Am. Chem. Soc., 2015, 137, 12045-12049. 